\title{
Development of an Automated Film-Reading System for Ballistic Ranges
}

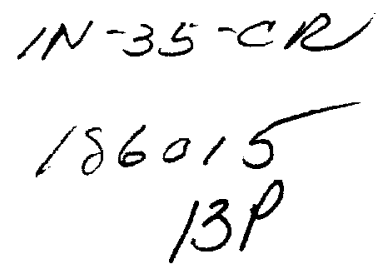

\author{
Leslie A. Yates* \\ Eloret Institute, Palo Alto, California 94303
}

\begin{abstract}
Software for an automated film-reading system that uses personal computers and digitized shadowgraphs is described. The software identifies pixels associated with fiducial-line and model images, and least-squares procedures are used to calculate the positions and orientations of the images. Automated position and orientation readings for sphere and cone models are compared to those obtained using a manual film reader. When facility calibration errors are removed from these readings, the accuracy of the automated readings is better than the pixel resolution, and it is equal to, or better than, the manual readings. The effects of film-reading and facility-calibration errors on calculated aerodynamic coefficients is discussed.
\end{abstract}

\section{Introduction}

Aeroballistic ranges, such as the Hypervelocity Free-Flight Aerodynamic Facility (HFFAF) at NASA Ames Research Center, provide invaluable data for flow-physics studies and for verification of computational fluid dynamics codes. These facilities can be operated over a wide range of Reynolds numbers, Mach numbers, and enthalpies, and they have two advantages over other types of ground-based facilities: 1) the freestreams are known and uncontaminated, and 2) the models are in free-flight; hence, there are no sting effects and the base flows are correct.

Aerodynamic, flowfield, radiation, and impact studies can all be conducted in ballistic range facilities. For these studies, model positions and orientations must be known. In the HFFAF, as well as in many other facilities, these measurements are obtained from a series of orthogonal view shadowgraphs that are taken at known times as the model flies down the range. An example of a shadowgraph which has been digitized is shown in Fig. 1. Recorded in these shadowgraphs are silhouettes (images) of the fiducial lines and model. The relative positions and orientations of these images are found by "reading" the shadowgraphs. Since the locations of the fiducial lines in the ballistic range are known, the position and orientation of the model image can be transformed into the range coordinate system.

The accuracy of these measurements affects the quality of the final results, particularly in the case of aerodynamic studies. Aerodynamic coefficients are determined by fitting calculated trajectories to model position and orientation measurements. ${ }^{1}$ In recent studies, measurement errors have limited the quality of the aerodynamic results in at least two cases: a revisitation of Pioneer-Venus ballistic range data with a Mars mission application ${ }^{2}$ and a study of the Aeroassist Flight Experiment trim angle. ${ }^{3}$ In the first case, the measurement errors for some of the runs were on the order of the lift induced swerve motion, and the lift coefficient could not be determined. In the second case, large errors in the position measurements limited the accuracy of the lift coefficient.

Large measurement errors can also mask deficiencies in the functions used to model the aerodynamic coefficients. If the modeling functions do not provide good descriptions of the aerodynamic coefficients, the difference between the calculated and measured trajectories has a functional dependency. This dependency can be used to check the validity of the aerodynamic modeling only if the measuramant orrnrs are less than the errors introduced by the modeling function.

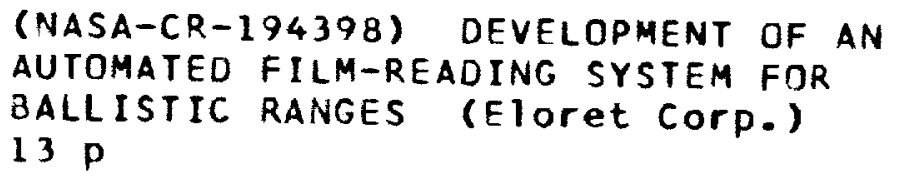

N94-13815

Unclas 
The model position and orientation measurements have two sources of error: film reading and the facility calibration of the fiducial system. In the past, shadowgraphs have been read manually, and the readings have been subject to both human interpretation and error. Furthermore, the vast majority of information recorded in the shadowgraphs is ignored; only points and edges of the model image are used to define its position and orientation.

The facility-calibration errors are predominantly controlled by inaccuracies in the measured locations of the fiducial lines in the ballistic range. When these errors are present, the residuals for the fit of the calculated trajectory to the actual trajectory are station dependent. These errors can be reduced by maintaining a history of the residuals and by adjusting the facility calibration when patterns are observed. ${ }^{4}$ Delays in updating the facility calibration are caused by the time requirements for reading film. With manual methods, film reading for a given run typically is not completed until days, if not weeks, after the run. It is unlikely that results will be obtained and assessed during the test. Hence, daily checks on the fiducial system are not possible, and days of testing may be wasted if the fiducial system is out of alignment.

In this paper, software for an automated film-reading system that uses digitized shadowgraphs and personal computers is described. Unlike the manual methods, this software uses all of the available information, and the accuracy and consistency of the film-reading measurements are improved. Furthermore, the personnel time and training requirements for film reading are reduced. Film reading for a shot can be completed within a day, and timely adjustments to the facility calibration and fiducial system are possible. The effect of the facility calibration on position measurements and aerodynamic results will be demonstrated using both automated and manual film readings.

\section{Automated Film Reading}

In the prototype system, a 300 pixels per inch, 256 gray-scale, flatbed scanner (Hewlett Packard ScanJet Plus) is used to digitize the shadowgraphs, and a Macintosh IIx is used for processing the digitized images. Typically, a 3 in. $\times 4$ in. portion of the shadowgraph contains all of the information necessary for determining the model position and orientation, and the memory requirements for storing one digitized shadowgraph is on the order of a megabyte.

The software used for manipulating the digitized shadowgraphs is a public domain program, Image, ${ }^{5}$ that has been modified to include procedures for identifying the fiducial-line and model images as well as techniques for calculating their positions and orientations. Once the shadowgraph has been digitized, the program displays it on the monitor, and the operator indicates points on each fiducial line and on the model to be used as starting points for the search routines. The search routines then identify all of the pixels associated with each fiducial line and with the model, and this pixel information is used to define the positions and orientations of the fiducialline and model images. These measurements are stored in a file, and after all of the shadowgraphs for the run have been read, they are transformed into the range coordinate system. The techniques for identifying the line and model positions will be discussed in the subsequent sections.

\section{Identification of the Fiducial-Line Location}

The searching routines that identify the pixels associated with any of the fiducial lines or with the model use the gray-scale information contained in the digitized images. In Fig. 2 grayscale values are plotted along a line one pixel wide (line $\mathbf{A}$ ) which is perpendicular to the fiducial lines. Definite peaks indicate the location of the fiducial-line images. Using this gray-scale information, the fiducial lines are presently defined by one of two methods. The first method simply searches a limited region approximately perpendicular to the fiducial line and determines which pixels fall within a given gray-scale range. The second method searches a limited region 
approximately perpendicular to the fiducial line for zeroes in the second derivative of the grayscale plot (indicated in Fig. 2 by the points B), and the edges of the fiducial lines are defined by these zeroes. A criterion on the magnitude of the second derivative on each side of the zero is used to prevent zeroes that are caused by background noise from being identified with the edge of the line.

In many shadowgraphs there is variation in the image exposure; for example, the top of the shadowgraph may be darker than the bottom. When this occurs, the gray-scale values defining the fiducial line vary along the length of the line, and the first method will define only a portion of the line (Fig. 3a). However, the second-derivative method will define the entire length of the line (Fig. $3 b$ ). With the second-derivative method, more information concerning the fiducial line is obtained, and the accuracy of its calculated location is improved. The second-derivative method is therefore chosen as the default line-identification method.

Identification of the entire line uses a marching procedure. First, the operator identifies a starting point on the fiducial line. Gray-scale values along a line perpendicular to the fiducial line (for example along line $\mathbf{A}$ in Fig. 2) are obtained, and the line-identification procedure uses either the gray-scale or second-derivative method to find the width of the fiducial line at this point. The routine then moves one pixel along the fiducial line, and the process is repeated. In this manner, the pixels that are associated with the fiducial-line image are identified. These pixels are shown in Figs. $3 a$ and $3 b$ by the areas highlighted in white.

Difficulties in this marching procedure can be caused by the superposition of images of scratches or gouges in the windows or of the model image itself onto the fiducial-line images. This superposition can appear to broaden the fiducial line or may cause the line-identification procedure to follow noise or scratches instead of the fiducial line.

Two methods are used to prevent the inclusion of unwanted pixels by the line-identification procedure. First, the average width of the line is calculated and continually updated. If any identified segment of the line is too broad or too thin, the pixels that define that segment are not included.

Second, the equation

$$
\mathrm{y}=\mathrm{mx}+\mathrm{b}
$$

is fit to the segments of the fiducial line which already have been identified. This equation is then extrapolated to a new segment of the fiducial line, and the pixels associated with this new segment are identified. If the identified pixels do not agree with the extrapolated position of the new segment or if the width of the newly defined segment does not agree with the average width of the line, the pixels are discarded. For example in Fig. $3 \mathrm{~b}$ gaps occur in the pixels identified with the fiducial lines when the width of the line or the center of the line does not agree with the predictions. By continually updating the equation for the line, the location of the next segment can be predicted, the search for pixels can be centered about this prediction, and new segments of the line can be identified even if they are separated from the previous segment.

Once the pixels associated with the fiducial line are identified, a weighted, least-squares method is used to calculate the slope and intercept for the line. The equations for the slope (m) and intercept (b) are

$$
\left(\begin{array}{cc}
\Sigma \omega_{i} & \Sigma \omega_{i} x_{i} \\
\Sigma \omega_{i} x_{i} & \Sigma \omega_{i} x_{i}^{2}
\end{array}\right)\left(\begin{array}{c}
b \\
m
\end{array}\right)=\left(\begin{array}{c}
\Sigma \omega_{i} y_{i} \\
\Sigma \omega_{i} x_{i} y_{i}
\end{array}\right)
$$

where the summations are over all of the pixels identified with the line, and the weighting function, $\omega_{i}$, is a function of the fraction of the pixel covered by the line. In Fig. 4 an example demonstrating the effect of finite pixel size on the shade of the individual pixels is shown. If the 
pixels are infinitesimal, there is a sharp delineation between the image and background, and all of the pixels associated with the image are the same color. If the pixels are finite, the edge of the image covers a fraction of the pixel. The shade of pixels at the edge of the image is somewhere between that of the interior points and the background shade, and it is proportional to the percentage of the pixel covered by the image.

For real shadowgraphs the fraction of the pixel covered by the fiducial line is a function of the difference between the pixel's shade and the background shade. In Eq. 2 the weights are simply given by this difference. The background color has a constant value if gray-scale values are used to define the line edge. A local value of the background color is used for the second-derivative method. This local value is the average gray-scale value just outside the defined boundary of the line. Irrespective of the definition of the background color, the pixels near the center of the fiducial line are typically much lighter than the background color and are weighted more heavily. The accuracy of the line location is greatly improved by using these weighting functions.

\section{Identification of the Model Location}

The pixels in the digitized shadowgraph that are associated with the model image are identified using shade. The model images cover a much smaller region of the shadowgraph than the fiducial lines, and variations in background exposure usually do not cause difficulties. However, a filter has been developed that minimizes variations in exposure, and this filter can be used when problems do occur. Methods which handle cases where the model image intersects images of scratches, gouges, or fiducial lines or where there is an attached shock have not yet been included in the film-reading software.

Once the pixels associated with the model image are identified, the centroid method ${ }^{6}$ is used to calculate its position and orientation. The centroid method can be used for any model image that has a symmetry axis. It uses a weighted, least-squares procedure to find the center of the model image and the orientation of the symmetry axis. The equations for the center $\left(x_{c}, y_{c}\right)$ and orientation $(\theta)$ are

$$
\begin{gathered}
\mathrm{x}_{\mathrm{c}}=\frac{\sum \omega_{\mathrm{i}} \mathrm{x}_{\mathrm{i}}}{\sum \omega_{\mathrm{i}}} \text { and } \mathrm{y}_{\mathrm{c}}=\frac{\sum \omega_{\mathrm{i}} \mathrm{y}_{\mathrm{i}}}{\sum \omega_{\mathrm{i}}} \\
\frac{1}{2} \tan (\theta)=\frac{\sum \omega_{\mathrm{i}}\left(\mathrm{x}_{\mathrm{i}}-\mathrm{x}_{\mathrm{c}}\right)\left(\mathrm{y}_{\mathrm{i}}-\mathrm{y}_{\mathrm{c}}\right)}{\sum \omega_{\mathrm{i}}\left[\left(\mathrm{x}_{\mathrm{i}}-\mathrm{x}_{\mathrm{c}}\right)^{2}-\left(\mathrm{y}_{\mathrm{i}}-\mathrm{y}_{\mathrm{c}}\right)^{2}\right]}
\end{gathered}
$$

The weighting functions are identical to those used for the fiducial lines. The center measurements define the center of the image and not the center of mass for the model. Therefore, a known translation must be applied to these measurements.

\section{Film-Reading Measurements}

Both simulated images and real shadowgraphs were used to test the film-reading software. In this section, the results are presented for simulated fiducial-line and cone images, for a scanner calibration using digitized parallel lines, and for actual shadowgraphs taken in the HFFAF.

\section{Simulated Images}

Straight lines that were computer generated and rotated by a known angle were used to represent digitized fiducial lines. Examples of a sharp line, a fuzzy line, and a fuzzy line with noise are shown in Fig. 5. In all three cases the film-reading software accurately identified pixels 
associated with the line, and in the case with noise, it ignored segments of the line which could not be separated from the noise. The calculated angle for all three cases was $2.00^{\circ}$; the accuracy of these readings was better than $0.005^{\circ}$. These measurements agreed with the known rotation, and the calculated intercepts were within 0.01 pixels of the known intercept. By using all of the pixels identified with the lines and weighted, least-squares procedures to calculate the slope and intercept, the resulting measurements were two orders of magnitude better than the pixel resolution.

Computer generated isosceles triangles were used to represent the images of cones. These representations were rotated through known angles and a smoothing algorithm was used to shade the edges. In Fig. 6 low and high resolution simulated images are shown. In both cases the filmreading software accurately identified the pixels associated with the cone images. The centroid method calculated the orientation of the cone as $9.99^{\circ}$ for the high resolution image; $10.04^{\circ}$ for the low resolution image. The actual value was $10.00^{\circ}$. These measurements are not as good as those for the simulated fiducial-line images. The simulated cone images are much shorter than the simulated line images, less information is available, and the calculated orientation of the cone is therefore less accurate. However, the errors are less than the nominal errors for the manual method, $\pm 0.1^{\circ}$.

\section{Scanner Calibration}

Repeatable errors in the film-reading measurements were introduced by the scanner. To minimize these errors, the scanner was calibrated using the following procedure. First, two parallel lines were created on a single sheet of paper using a laser printer. This sheet was then placed at several locations on the flatbed scanner and digitized. The slope and intercept for each line were then calculated using the software developed for the fiducial lines, and the distance between the two lines was determined. The results are shown in Fig. 7.

The scanning unit of the Hewlett Packard ScanJet Plus is a horizontal unit that moves vertically down the page, and although the scanner is nominally 300 pixels per in., the widths and lengths of the scanned sections represented by each pixel are not constant. The variations in the measured distances in Fig. 7 are functions of the differences in these widths and lengths. Initial studies indicated that the widths of these sections are independent of the vertical location (within desired accuracy) and the lengths are independent of the horizontal location.

In Fig. 7a the distance between two vertical lines is shown. The measured distance varies smoothly across the scanner, and the differences in the measurements are much smaller than the pixel size, on the order of \pm 0.05 pixels. Hence, the width of the pixels is nearly constant. Although the difference in pixel widths is small, it is not random, and it can accumulate and affect the filmreading measurements. A function has been included in the film-reading software that accounts for this variation.

The variation in the vertical height of the pixels, represented by the variation in distance between two horizontal lines (Fig. 7b), is not small and does not vary smoothly. This is the direction that the scanning unit moves, and it is not surprising that errors in this direction are larger. However, repeated tests have indicated that the distance measurements are repeatable, and a lookup table is used to correct the measured locations and distances.

\section{Digitized Shadowgraphs}

The film-reading software was used to read fifteen side and fifteen bottom shadowgraphs for a single calibration shot in the HFFAF. For this calibration shot, a sphere model was used. The model velocity was $1.8 \times 10^{4} \mathrm{ft} / \mathrm{sec}$, and the test section pressure was less than $5 \times 10^{-5} \mathrm{~atm}$. The aerodynamic forces were negligible when compared to gravitational forces, and the model followed a parabolic trajectory. In addition to being read by the automated film reader, the 
shadowgraphs were read twice manually. The deviation of all three readings from the calculated trajectory is plotted in Fig. 8.

In the down-range direction (the $\mathrm{x}$-direction) there is some deviation of all three readings from zero - the calculated and experimental trajectories do not coincide. This deviation is the result of facility calibration errors. However, there is excellent agreement between all three readings, and any differences between the automated and manual results are on the order of the differences between the two manual readings.

In the vertical direction (z-direction), the agreement between the automated and manual readings is not as good. This is a direct result of the difficulty in calibrating the scanner in the vertical direction; a more careful calibration in this direction should improve the results to the desired accuracy.

The horizontal swerve measurements (y-direction) show undesirably large variations between the automated and manual readings and between the two manual readings. This is due to the poor quality of the shadowgraphs for the bottom stations: the images are often very light with little contrast, and properly defining the fiducial lines using either the automated or the manual film reader is difficult. It should be noted, however, that when the two manual readings are in close agreement, the automated reading is also in close agreement. In addition, the root mean squares (rms) of the residuals for the automated readings are generally smaller than the rms values for the manual readings.

For this sphere shot, the automated film reader took far less time than the manual method. The entire shot, all thirty shadowgraphs, was read in approximately three hours. This time represents a sizable reduction from the eight or more hours required for the manual film reader, and it should be independent of the model shape. A major portion of the three hours was dedicated to digitizing the shadowgraphs; this included the time required for placing the shadowgraphs on the scanner, previewing the digitized image, selecting the essential portion of the image, making necessary contrast adjustments, and digitizing the final image. If digital cameras are used to take the shadowgraphs, as is planned at the free-flight facility of the Air Force Wright Laboratory Armament Directorate (Eglin Air Force Base), ${ }^{7}$ the time requirements for reading film could be reduced even further. Searching for the pixels associated with the fiducial-line and model images and then using least-squares methods to calculate their positions and orientations took less than a minute for good images. This process took longer for images with poor contrast since the search procedures had difficulties in defining the fiducial lines or models and parameters had to be changed.

To further assess the capabilities of the film-reading software, shadowgraphs of a blunt cone were digitized and read. These readings were then compared to manual readings. In Fig. 9 representative angular and position measurements are shown. The rms values for the fit of the calculated trajectory to the manual and automated angular measurements are approximately the same: $0.22^{\circ}$ for the manual measurements, $0.18^{\circ}$ for the automated readings. The position measurements are improved by almost a factor of two; the rms for the manual measurements is $0.0067 \mathrm{in}$.; for the automated readings, $0.0037 \mathrm{in}$.

It should be noted that there is approximately a factor of two difference in scale between the shadowgraphs and the range measurements; hence, the film-readings are accurate to $0.0019 \mathrm{in}$. or 0.6 pixels. This is much larger than the few hundredths of a pixel obtained for the digitized parallel lines. The rms values for the digitized shadowgraph readings are larger for a variety of reasons. One source of error is related to the quality of the shadowgraphs. In shadowgraphs with poor contrast, pixels associated with the fiducial-line and model images are difficult to identify. Furthermore, there are often scratches or gouges in the windows, and the images of these imperfections intersect the model image. If the model identification procedure does not identify all of the pixels associated with the model image or if it includes extra pixels, there will be measurement 
errors. In this case, the model image could generally be separated from the shock, and the modelidentification procedure did not include pixels associated with the shock.

The remaining sources of errors include the calibration of the scanner and the calibration of the facility. The difficulties caused by nonuniform pixel size have been discussed previously. Facility calibration errors can be caused by errors in the measured locations of the fiducial lines or by the shadowgraph system. One type of shadowgraph induced error is caused by the shadowgraph light source. In the transition between shadowgraph readings and range measurements, a collimated light source is assumed. However, the images of four plumb fiducial lines in the side-view shadowgraphs are not parallel, thus indicating that the light is not perfectly collimated. This will result in small errors in the measured model position. Facility-calibration errors and methods for minimizing these errors are discussed in the following section.

\section{Facility-Calibration Errors}

Over twenty years ago, Chapman ${ }^{4}$ suggested techniques for tracking and correcting errors caused by inaccuracies in the measured locations of fiducial lines (or marks). Although these techniques can sizably reduce errors in model position and orientation measurements and, therefore, improve the aerodynamic results, efforts to regularly update facility calibrations during testing have been minimal. Measurements obtained using the film-reading software provide an excellent example as to the importance of facility calibration.

In Fig. 10 residuals for three calibration shots are shown. Spheres were used for all three shots, and the test section pressures were on the order of $5 \times 10^{-5} \mathrm{~atm}$. The measurements were made using the film-reading software. The residuals are much larger than the desired one or two thousandths of an inch. The pattern in the residuals from one station to the next appears to be random. However, at each station the residuals are clustered about a single value and are not random. Hence, the source of the errors is the facility calibration and not the film reading.

A random pattern in the residuals from one station to the next is expected in the down range direction; the fiducial system in this direction consists of individual vertical wires at measured intervals. In the $y$ - and $z$-directions, however, the fiducial lines are single catenary wires strung the entire length of the range. Smooth corrections were anticipated; these would represent corrections to the catenary wire equations that had been used to define the position of the wires at each station. However, the corrections in the y-and z-directions do not follow a smooth pattern, and some other factor, such as kinks along the entire length of the catenary wire or the non-parallel nature of the light sources, must be contributing to the errors.

The effects of errors in the facility calibration were removed by averaging the residuals for these three calibration shots and then correcting the measurements at each station by the average value. The residuals for the corrected measurements are shown in Fig. 11. The rms value for the down range direction $(x)$ has been reduced from 0.0079 to $0.0013 \mathrm{in}$.; for the horizontal swerve direction (y), from 0.0058 to 0.0019 in.; and for the vertical direction ( $\mathrm{z}$ ), from 0.0040 to $0.0011 \mathrm{in}$. Since there is a multiplication factor of two between the film and range coordinates, the rms values for the film-reading errors range from 0.0005 to 0.001 in.; this is better than one third pixel. It should be noted that at these velocities, the model travels approximately 0.001 in. during 5 nanoseconds, the timing uncertainty for the HFFAF.

The accuracy of the calculated aerodynamic coefficients is a function of both the measurement and modeling errors. The measurement errors, such as the film-reading and facility-calibration errors, can be reduced by improving the measurement techniques and by constantly updating the facility calibration. The modeling errors are caused by inadequate mathematical representations of the aerodynamic coefficients, and their contribution to the residuals will have a definite pattern. If the measurement errors are small, the modeling errors can be detected from this pattern. If the measurement errors are large, they mask the pattern, the residu- 
als cannot be used to check the modeling functions, and other methods must be used to check the validity of the model. If the measurement errors are too large, it may be necessary to assign or neglect higher order terms in the aerodynamic coefficient expansions. This is particularly important for trim-angle and lift-coefficient measurements when experimental data are limited. ${ }^{3}$

In some cases the measurement errors are so large that the aerodynamic coefficients cannot be determined even if the appropriate modeling function is known. Generally, drag and moment forces have large effects on the trajectory of the vehicle, and the measurement errors are much smaller than the drag or moment induced motion. However, lift forces may have only a small effect on the motion, and this small effect can be masked by film-reading or facilitycalibration errors.

Ballistic range tests of a Pioneer-Venus model which were conducted in the HFFAF provide an excellent example as to the difficulties that are encountered when measurement errors are on the order of the lift induced swerve. The Pioneer-Venus model is axially symmetric, and the swerve motion is approximately equal to a constant times the angular motion. In Fig. 12 the swerve and angular motions for a single run are plotted. The velocity for this shot was $1.1 \times$ $10^{4} \mathrm{ft} / \mathrm{sec}$, the test gas was air, and the test section pressure was $1 \mathrm{~atm}$. The angular motion (dashed line) is approximately sinusoidal with an amplitude less than $2.5^{\circ}$. When the errors in the facility calibration are not removed, the peak amplitude of the swerve motion (dotted line) is less than 0.02 in. It does have some sinusoidal characteristics. However, its amplitude and offset from zero are constantly changing, and it does not closely follow the angular motion. For this case, the calculated lift coefficients will not be accurate.

In Fig. 13 the residuals in the swerve direction are shown for several free-flight tests of Pioneer-Venus models at several different freestream conditions. The shadowgraphs for these shots were read manually. The residuals are large, and at each station they are clustered about a nonzero value. The facility calibration can be corrected by subtracting the average of the residuals at each station from the swerve measurements. These corrections range from -0.011 to 0.012 in., and they are large compared to the amplitude of the swerve motion.

Once the corrections for the facility calibration are made (solid line in Fig. 12), the swerve motion is sinusoidal. It closely approximates the angular motion, and the calculated lift coefficient will be more accurate. For the uncorrected data, the lift coefficient was calculated to be $0.35 \pm 0.10$. For the corrected data, the lift coefficient was $0.21 \pm 0.05$. The calibration errors have a significant effect on the lift coefficient and its predicted error. By removing the facility-calibration errors, it is possible to calculate lift coefficients for lift induced motions of a few hundredths of an inch. Minimizing the film-reading errors will further improve the orientation and position measurements, and accurate aerodynamic coefficients can be found for even smaller amplitude motions.

\section{Concluding Remarks}

Software for an inexpensive (total hardware costs are less than $\$ 12 \mathrm{~K}$ ) film-reading system that uses digitized shadowgraphs and personal computers has been developed and undergone preliminary testing. This software identifies pixels associated with the fiducial-line and model images, and it uses weighted, least-squares techniques to calculate the image positions and orientations. Techniques have been developed that differentiate between the fiducial-line images and noisy data. The accuracy of the automated readings is better than the pixel resolution, and it is equal to, or better than, the accuracy of the manual readings. The time required for film reading has been reduced, and preliminary results can now be obtained within a day of the run. Hence, daily checks of the fiducial system are possible, and days of testing will not be wasted if the fiducial system is out of alignment. 
Future additions to the film-reading software will include methods that use the second derivative of the gray-scale plots to define the pixels associated with the model image. Methods to differentiate between the model image and noisy data will also be developed and implemented. These two additions to the software involve only the model identification procedures. They are independent of the method used to calculate the position and orientation of the model image, i.e., the centroid method. As formulated, the centroid method cannot be used for models which do not have an axis of symmetry in their two-dimensional projection. It is often difficult to manually measure the position and orientation of these types of models, and new methods that will accurately measure the position and orientation of three-dimensional models must be developed.

By reducing both the film-reading and facility calibration errors, the quality of ballistic range results can be improved. The film-reading errors can be reduced by using computers to reduce digitized shadowgraphs to position and orientation measurements. The facility calibration errors can be minimized by tracking and correcting errors caused by inaccuracies in the measured fiducial-line locations. With these reductions in the measurement errors, accurate aerodynamic coefficients for very small amplitude motions will be possible.

\section{Acknowledgments}

Support for Leslie A. Yates was provided by NASA Grant NCC 2-583. This work was part of a cooperative program between NASA Ames Research Center and the Air Force Wright Laboratory Armament Directorate.

\section{References}

${ }^{1}$ Chapman, G.T. and Kirk, D.B., "A New Method for Extracting Aerodynamic Coefficients from Free-Flight Data," AIAA Journal, Vol. 8, no. 4, April 1970, pp. 753-758.

2Packard, J.D., Griffith, W.C., Yates, L.A., and Strawa, A.W., "A Ballistic Investigation of the Aerodynamic Characteristics of a Blunt Vehicle at Hypersonic Speeds in Carbon Dioxide and Air," AIAA Paper 92-0328, Jan. 1992.

${ }^{3}$ Yates, L.A., and Venkatapathy, E., "Free-Flight Trim-Angle Predictions for the Aeroassist Flight Experiment," Journal of Spacecraft and Rockets, Vol. 29, May-June 1992, pp. 335-343.

${ }^{4}$ Chapman, G.T., "Aerodynamic Parameter Identification in Ballistic Range Tests," Proceedings of the 1972 Army Numerical Analysis Conference, ARO-D Report 72-3, U.S. Army Research Office, Durham, North Carolina, 1972, pp. 73-109.

${ }^{5}$ Rasband, W., Users manual and source code for Image, Version 1.19, Oct. 1989. Software, source code and manual available via anonymous ftp from alw.nih.gov.

${ }^{6}$ Holt, D.M., "Proof-of-Principle High Speed Electronic Imaging System - Phase II," AFATLTR-85-065, Air Force Armament Laboratory, Eglin Air Force Base, Florida, Dec. 1987.

${ }^{7}$ Becker, B., Winchenbach, G.L., Snyder, D.R., Macke, H., and Rowe, W.J., "Upgrade to the Aeroballistic Research Facility and Image Analysis System and Film Reader," presented at the 42nd Meeting of the Aeroballistic Range Association, Adelaide, Australia, Oct. 1991. 


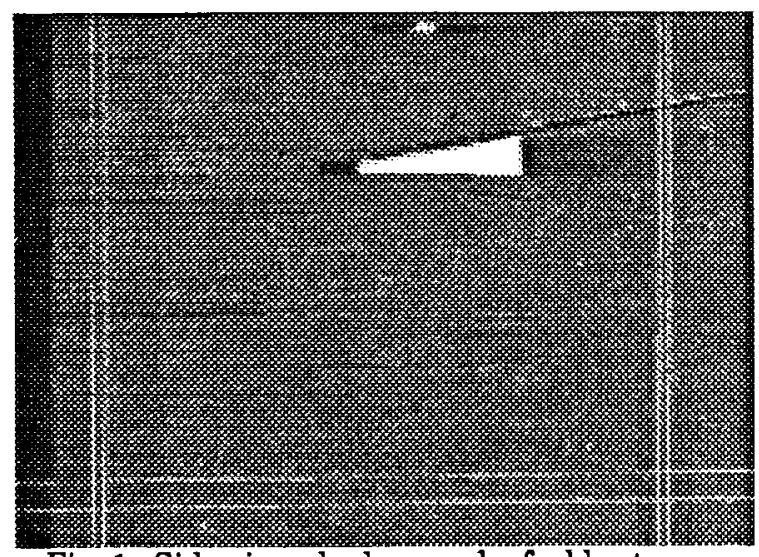

Fig. 1 Side-view shadowgraph of a blunt cone.
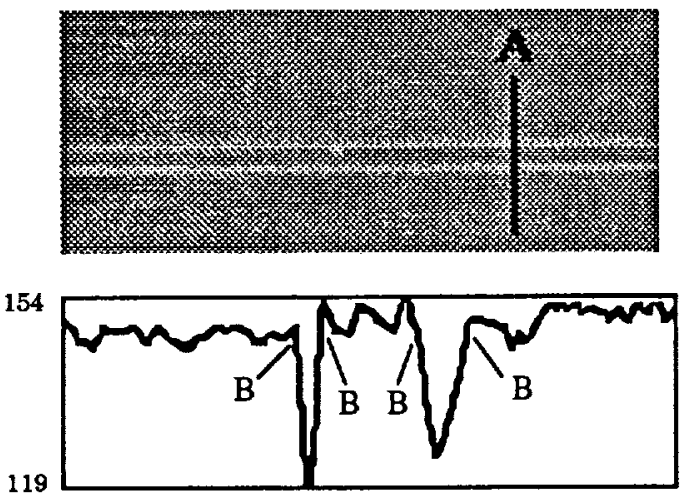

Fig. 2 Gray-scale variation across the fiducial line image.

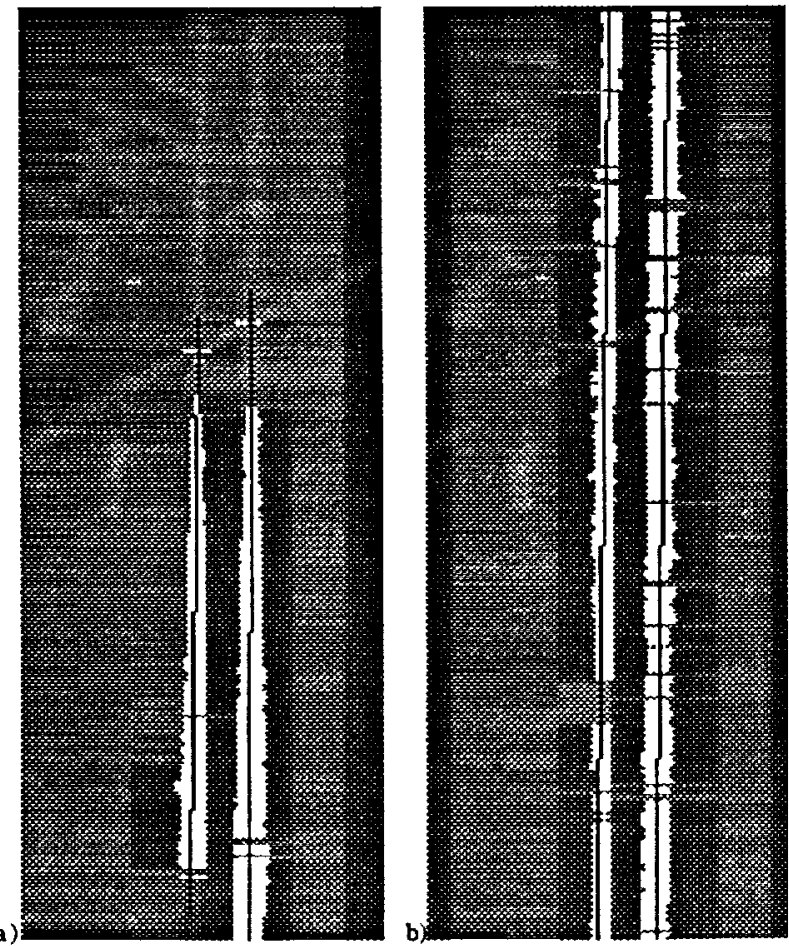

Fig. 3 Identification of the fiducial lines by a) shade, b) second derivative. White areas represent pixels identified with the fiducial lines.

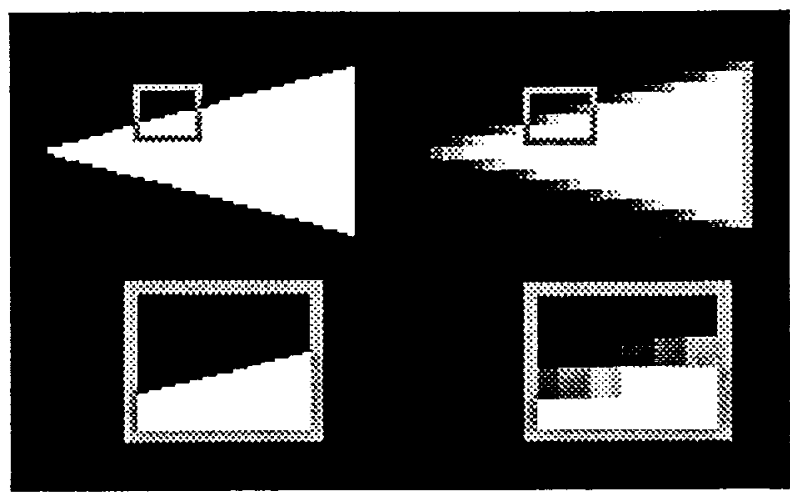

Fig. 4 Shading of edge pixels.

a)

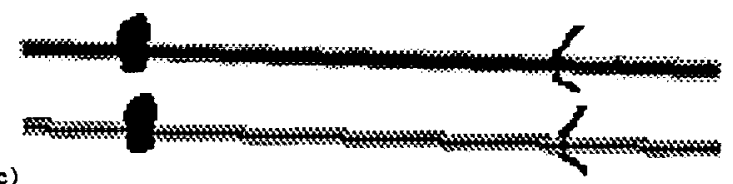

Fig. 5 Simulated lines. a) Sharp line. b) Fuzzy line. c) Fuzzy line with noise. Gray area represents pixels identified with the line, black line represents the calculated center.

a)
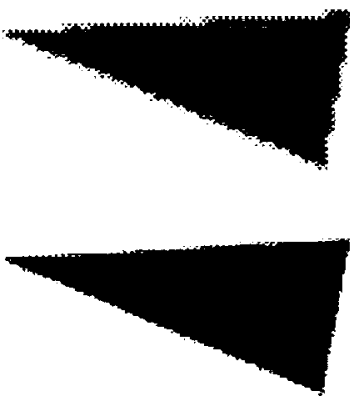

Fig. 6 Orientation for simulated cone image. a) Coarse resolution. b) Fine resolution. Gray represents pixels identified with the cone. 

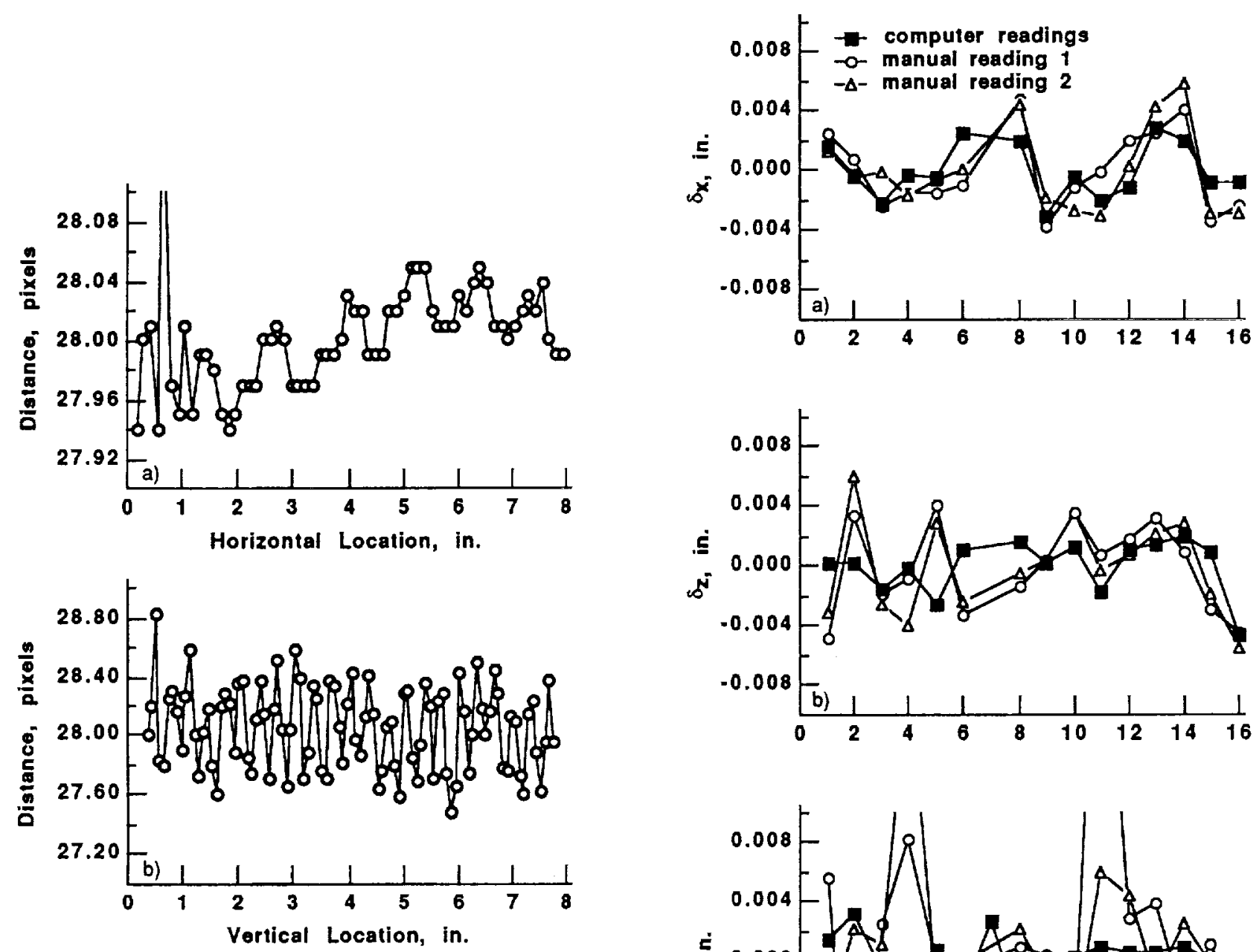

Fig. 7 Distance between two parallel lines. a) Vertical lines. b) Horizontal lines.
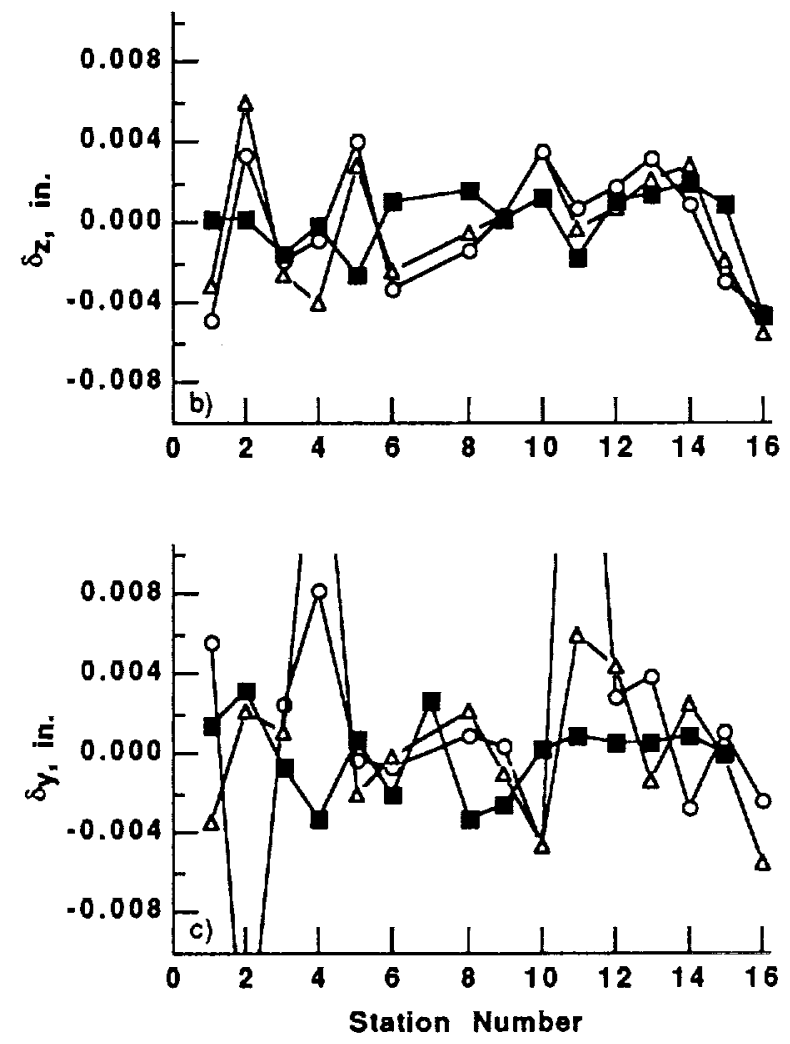

Fig. 8 Comparison of automated and manual readings. Side view: a) down-range, b) vertical. Bottom view: c) horizontal. 

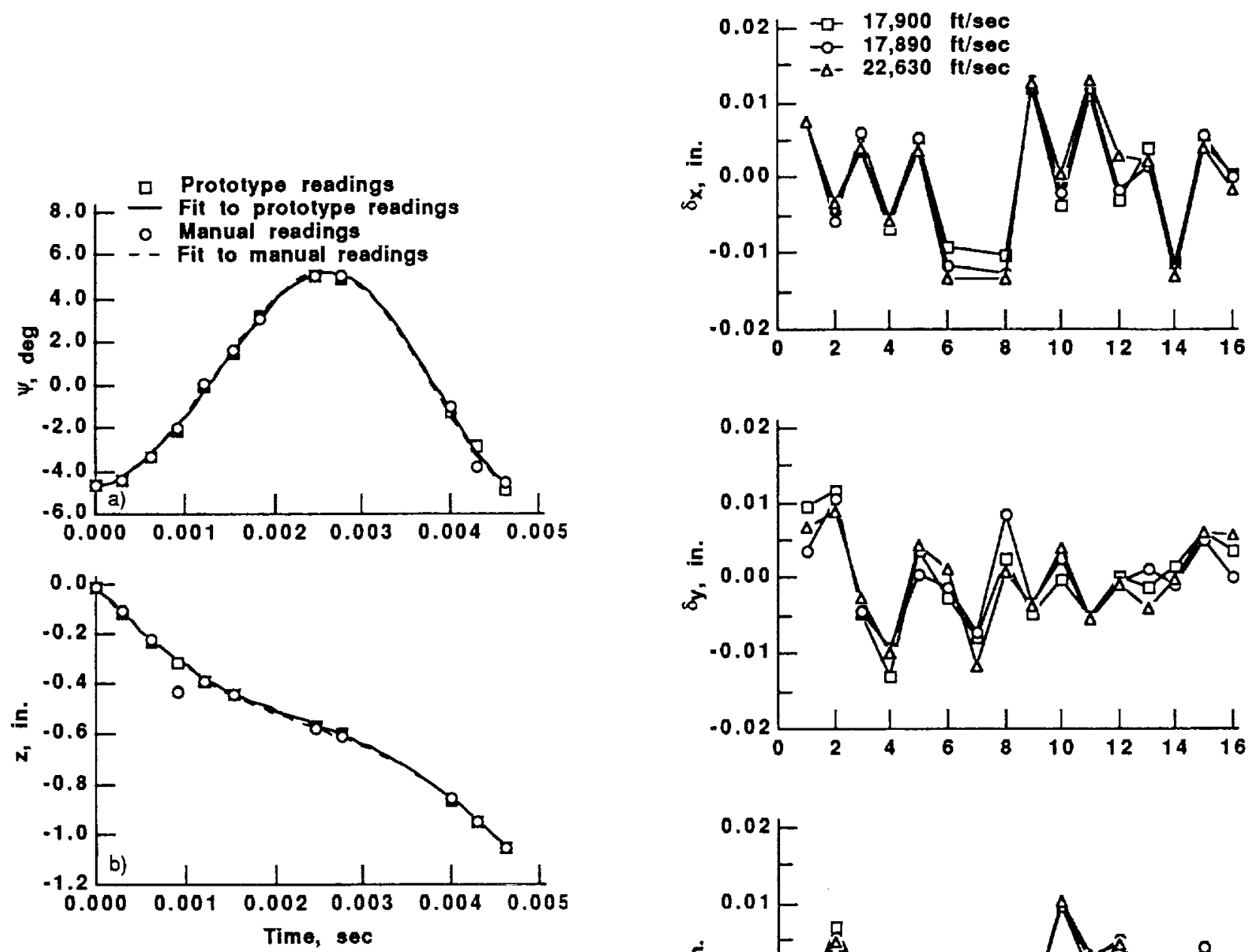

Fig. 9 Comparison of cone readings. a) Angle, $\psi . b$ ) Vertical displacement, $\mathbf{z}$.
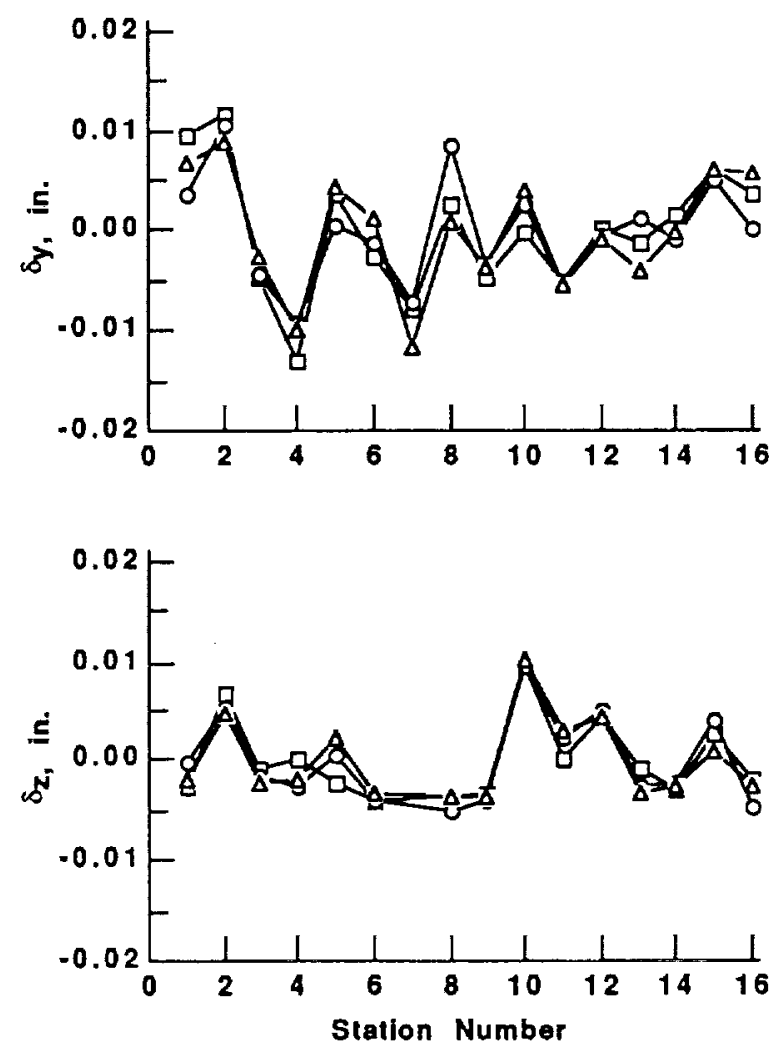

Fig. 10 Residuals. 

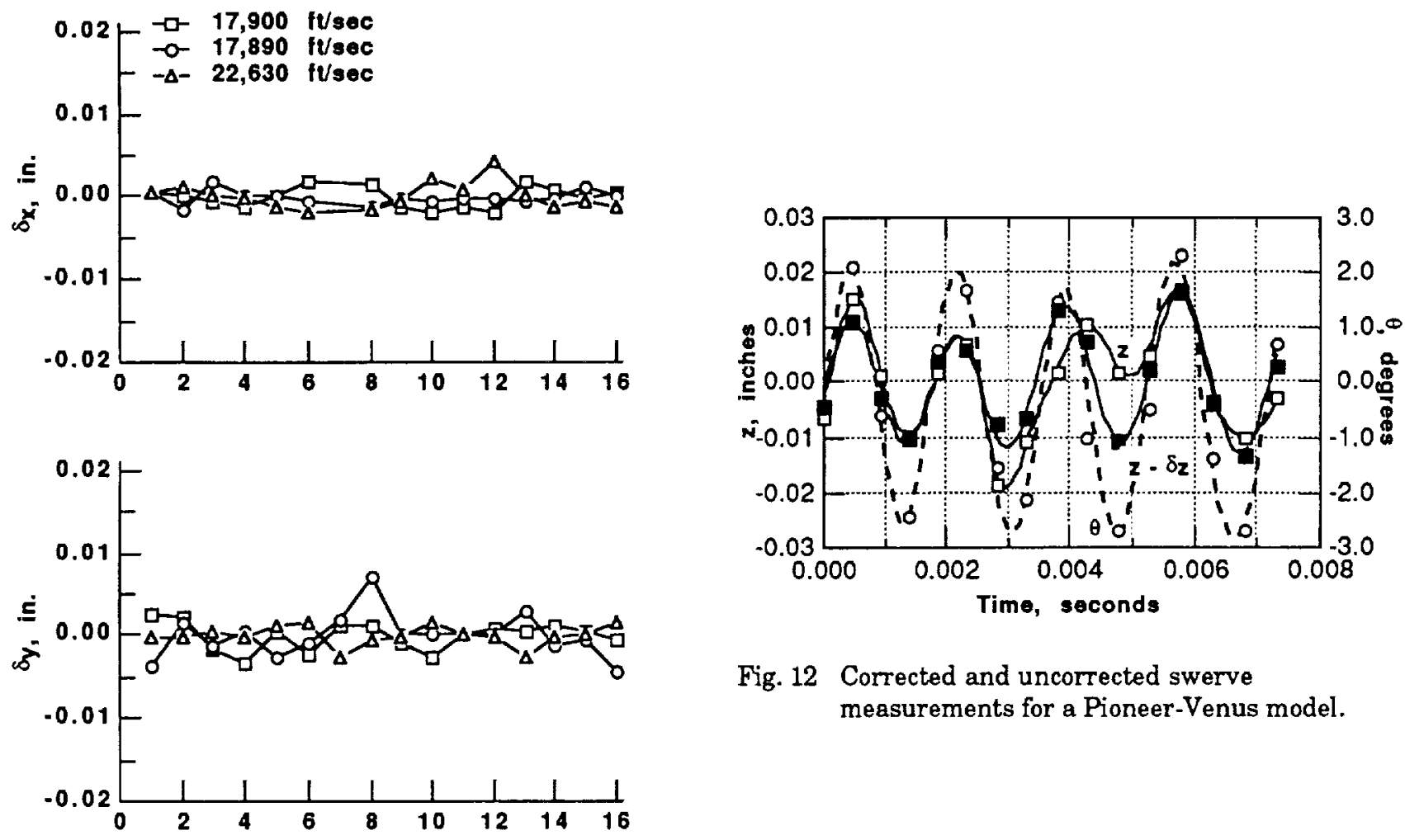

Fig. 12 Corrected and uncorrected swerve measurements for a Pioneer-Venus model.
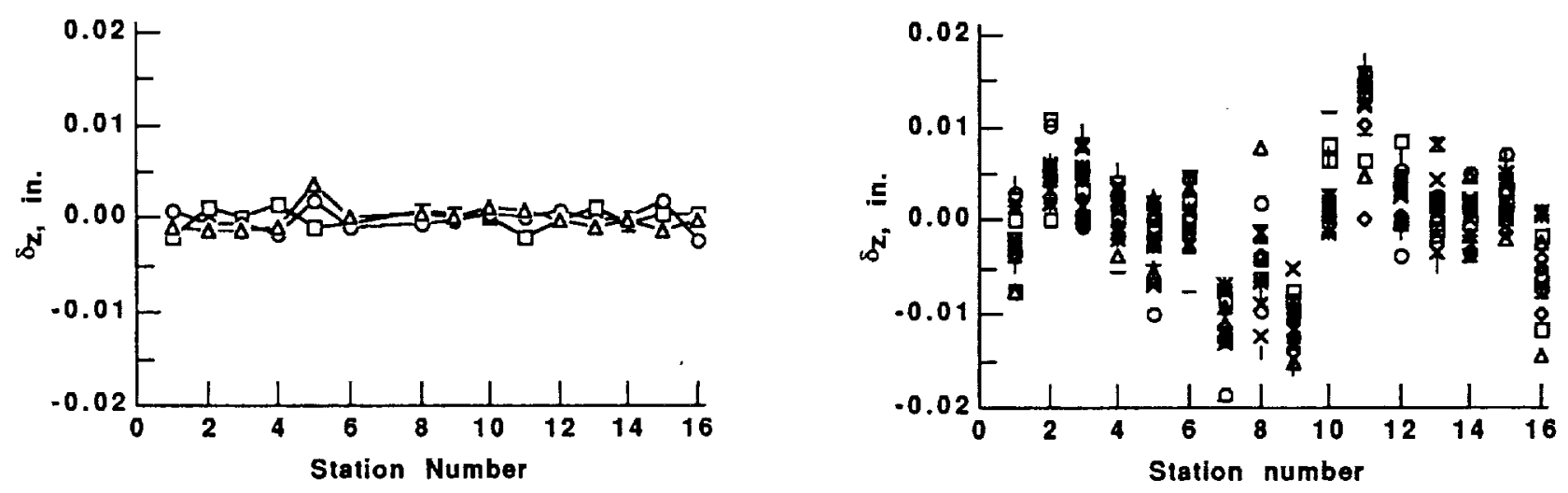

Fig. 11 Residuals after facility calibration.

Fig. 13 Residuals for a series of Pioneer-Venus tests in the NASA Ames ballistic range. 